\title{
Neutrino mixing and discrete symmetries
}

\author{
Bo $\mathrm{Hu} *$ \\ Department of Physics, Nanchang University, Jiangxi 330031, China
}

\begin{abstract}
In this paper we discuss a new way to derive neutrino mixing patterns, which originates from the idea proposed in a recent article by Hernandez and Smirnov. Its applications to various cases are discussed. We first present the complete set of possible mixing patterns for the minimal case where unbroken residual symmetries of the Majorana neutrino and left-handed charged-lepton mass matrices obey some general assumptions that are also satisfied by many models based on discrete symmetries. We find that they are either well-known mixing patterns or phenomenologically disfavored ones. It shows clearly that, for full-mixing matrices to fit the mixing data with small or negligible corrections, it is necessary to go beyond the minimal scenario. We present an explicit formalism for a rather general nonminimal case. Some applications and phenomenological implications are discussed. Several new mixing patterns are derived.
\end{abstract}

*Electronic address: bohu@ncu.edu.cn 


\section{INTRODUCTION}

The discovery of neutrino oscillations provides clear evidence for physics beyond the Standard Model (see, e.g. [1, 2] and for recent global fits, see [3 -5]). In this work we study neutrino mixing determined by underlying discrete flavor symmetries [6, 7]. Models based on this approach have been discussed extensively (see, e.g., [8 31] for some recent works) and they often lead to intriguing mixing patterns, including the well-known tribimaximal mixing (TBM) [32] and bimaximal mixing (BM) [33]. However, a challenge is posed by recent experimental results on the reactor mixing angle [34 38] because many models discussed previously did not predict the measured value of $\theta_{13}$ (see, e.g. [39] or [40] for a survey of model predictions). Although good agreement with the data can be achieved by introducing corrections or other means (see, e.g. [41 44]), it is still worthwhile to seek for symmetries and mixing patterns that are in good agreement with the data without introducing large corrections which may badly break the (would-be) residual symmetries, e.g. the THF mixing or bi-trimaximal mixing discussed in recent articles [45-47].

In this paper we focus on Majorana neutrinos. The mass matrix of Majorana neutrinos has a $\mathbf{Z}_{2} \times \mathbf{Z}_{2}$ symmetry, and the left-handed charged lepton mass matrix has a $U(1) \times U(1)$ symmetry [48, 49] if they are required to belong to $S U(3)$. When restricted to discrete symmetries, the latter is usually reduced to a $\mathbf{Z}_{m}$ or other finite symmetry belonging to the $U(1) \times U(1)$ (for some examples, see [45]). In models based on discrete symmetries, these symmetries may coincide with the symmetries preserved by relevant mass terms after the breaking of flavor symmetry, ${ }^{1}$ which is the case that will be considered in this paper. We follow [50] and carry out a model independent study of neutrino mixing. The basic assumption we adopt is that the residual symmetries of the mass matrices belong to an underlying discrete flavor symmetry. Our main concern is the general results that can be drawn from this assumption.

We find that the idea of [50] leads to a new way to derive mixing matrices, which is simpler than the group theoretical method. It does not require a flavor group to be specified in advance. On the contrary, it can provide the necessary conditions from which one can find the flavor symmetry corresponding to a mixing matrix or mixing-matrix column derived by

\footnotetext{
${ }^{1}$ Models with completely broken symmetries can be constructed (e.g., 55]), which are beyond the scope of this work and hence will not be considered here.
} 
this method. If the flavor symmetry is known, it can serve as a quick way to derive mixing matrices or elements and cross-check with group theoretical calculations. From the equation constraining the residual symmetries, an interesting result we find is that, under some general and plausible assumptions which are satisfied by many models and adopted in [50], one can prove rigorously that phenomenologically viable mixing patterns that discrete symmetries can lead to include only TBM, BM and the golden ratio mixings. It also provides a different way to approach some known results. For example, one can show that the minimal horizontal symmetry that can produce TBM as a full-mixing matrix is $\mathbf{S}_{4}$, which was first pointed out in [51].

As suggested by the no-go result mentioned above, those assumptions may have to be relaxed, and hence new mixing patterns can be obtained. Explicit formalism for a rather general nonminimal case is also provided in this paper. Several interesting applications are discussed and new mixing matrices are presented. Their phenomenological implications are also discussed briefly. We find that, although not impossible at all, it is still difficult to find a mixing matrix that can produce all the mixing data. This result agrees with [53] and [54] in which the results of the searches for groups up to certain large orders are reported.

Although our approach is based on that of [50], the concerns and discussions differ quite a lot. We also note that in a recent work [52], authors of [50] also generalize the results of their previous work. But the subjects and the formalism are still quite different. More attention is paid to partial mixings and phenomenology in [52], and their discussions are more group theoretical oriented. Most of our attention is concentrated on full-mixing patterns. A general result about the minimal scenario of [50] is presented and discussed in this paper. Besides the formalism that can be used in general cases, we also provide a simple formalism for a rather general nonminimal scenario from which most known mixing patterns, including those discussed in [52] or [45], can be derived. It also provides a different way to understand the permutation feature of some mixing patterns. Several new mixing patterns are also derived and presented.

The paper is organized as follows. In Sec. II, we review briefly the idea proposed in [50]. For later convenience, the formalism for squared mixing elements, which can be used in general cases, is presented in this section. In Sec. III, the attention is focused on the minimal scenario. A general result concerning possible mixing patterns is presented and discussed. Then, in Sec. IV, the consequences of relaxing some of the assumptions adopted 
in the minimal scenario and the relevant phenomenology are discussed. We summarize in Sec. V.

\section{NEUTRINO MIXING AND DISCRETE SYMMETRIES}

We first set up our notations. We denote the generator of the symmetry of the left-handed charged-lepton mass matrix (i.e., $M_{l}^{\dagger} M_{l}$ where $M_{l}$ is the mass matrix of charged leptons) by $T$ and those of the Majorana neutrino mass matrix by $S_{i}$ where $i=1,2$, or 3 . The flavor symmetry is denoted by $G_{f}$, and the symmetries generated by $T$ and $S_{i}$ are denoted by $G_{e}$ and $G_{\nu}$, respectively. In this work, we always assume that $G_{\nu}=\mathbf{Z}_{2} \times \mathbf{Z}_{2}$ and $T$ generates a $\mathbf{Z}_{m}$ symmetry. We also assume that $T$ and $S_{i}$ belong to $S U(3)$ and, for simplicity, $\operatorname{Tr}[T]$ is real in most of our discussions. Some comments on these assumptions are given at the end of this section.

In the basis where the charged lepton mass matrix is diagonal, $T$ can be written as a diagonal matrix denoted by $T_{d}$. In the case where $\operatorname{Tr}[T]$ is real, $T_{d}$ can be written as one of the following three matrices:

$$
\begin{aligned}
& T_{e} \equiv T_{1}=\operatorname{diag}\left\{1, e^{2 \pi i k / m}, e^{-2 \pi i k / m}\right\}, \\
& T_{\mu} \equiv T_{2}=\operatorname{diag}\left\{e^{2 \pi i k / m}, 1, e^{-2 \pi i k / m}\right\}, \\
& T_{\tau} \equiv T_{3}=\operatorname{diag}\left\{e^{2 \pi i k / m}, e^{-2 \pi i k / m}, 1\right\},
\end{aligned}
$$

since it belongs to $S U(3)$. Therefore,

$$
\operatorname{Tr}[T]=1+2 \cos 2 \pi k / m
$$

when $\operatorname{Tr}[T]$ is real. In addition, we assume that $T_{\alpha}(\alpha=e, \mu$, or $\tau)$ is nondegenerate or $m \geq 3$; otherwise charged lepton masses cannot be guaranteed to be nondegenerate and ambiguities in mixings may arise [49]. Further discussions can be found at the end of this section.

The key assumption on $T$ and $S_{i}$ is that they belong to $G_{f}$, a finite flavor symmetry. It then follows that $\left(S_{i} T\right)^{p_{i}}=\mathbb{I}$ for some $p_{i}$ which leads to $[50]^{2}$

$$
\left(S_{i} T\right)^{p_{i}}=\left(U_{P M N S} S_{i}^{d} U_{P M N S}^{\dagger} T_{\alpha}\right)^{p_{i}}=\mathbb{I}
$$

\footnotetext{
${ }^{2}$ Other relations are possible if $T$ or $S_{i}$ appear in $G_{f}$ indirectly.
} 
where $U_{P M N S}$ is the lepton mixing matrix and $S_{i}^{d}$ are diagonal matrices given by

$$
S_{1}^{d}=\operatorname{diag}\{1,-1,-1\}, \quad S_{2}^{d}=\operatorname{diag}\{-1,1,-1\}, \quad S_{3}^{d}=S_{1}^{d} S_{2}^{d} .
$$

Then, denote $\left(S_{i} T\right)^{-1}=T^{m-1} S_{i}$ by $W_{i}$. One finds that

$$
\left(W_{i}\right)^{p_{i}}=\left[\left(S_{i} T\right)^{-1}\right]^{p_{i}}=\mathbb{I} .
$$

Our basic assumption on the residual symmetries can then be summarized by the relations

$$
S_{i}^{2}=T^{m}=\left(W_{i}\right)^{p_{i}}=S_{i} T W_{i}=\mathbb{I}
$$

The characteristic equation of $W_{i}$ can be written as [50]

$$
\lambda^{3}+w_{i} \lambda^{2}-w_{i}^{*} \lambda-1=0
$$

where $w_{i}=-\operatorname{Tr}\left[W_{i}\right]$. Because of Eq.(15), one has $\lambda^{p_{i}}=1$, and from the equation above, it follows that if $w_{i}$ is real, the eigenvalues of $W_{i}$ are given by $\lambda=1, e^{2 \pi i n_{i} / p_{i}}$, or $e^{-2 \pi i n_{i} / p_{i}}$, and hence $w_{i}=-1-2 \cos 2 \pi n_{i} / p_{i}$.

Until now we have followed [50] in which more details can be found. Below we shall discuss first the general situation. Later in the next section we return back to the minimal scenario discussed in [50]. Since $\operatorname{Tr}\left[W_{i}\right]=\operatorname{Tr}\left[\left(S_{i} T\right)^{-1}\right]=\operatorname{Tr}\left[\left(S_{i} T\right)^{\dagger}\right]=\left(\operatorname{Tr}\left[S_{i} T\right]\right)^{*}$, one finds that

$$
w_{i}^{*}=-\left(\operatorname{Tr}\left[W_{i}\right]\right)^{*}=-\operatorname{Tr}\left[U_{P M N S} S_{i}^{d} U_{P M N S}^{\dagger} T_{d}\right]=\operatorname{Tr}\left[T_{d}\right]-2 \operatorname{Tr}\left[A_{i} T_{d}\right]
$$

where $A_{i}$ are defined as

$$
A_{i}=\operatorname{diag}\left\{\left|\left(U_{P M N S}\right)_{1 i}\right|^{2},\left|\left(U_{P M N S}\right)_{2 i}\right|^{2},\left|\left(U_{P M N S}\right)_{3 i}\right|^{2}\right\}
$$

Equation (8) is the most general formalism for mixing elements in this framework. As will be shown later, it can be written in more convenient and explicit forms.

If all $S_{i} \in G_{f}$, an important condition can be derived from Eq.(8). Summing over $i$ leads to

$$
\sum_{i=1}^{3} w_{i}^{*}=-\sum_{i=1}^{3}\left(\operatorname{Tr}\left[W_{i}\right]\right)^{*}=\operatorname{Tr}\left[T_{d}\right]
$$

which follows from $\sum_{i=1}^{3} S_{i}^{d}=-\mathbb{I}$ and the unitarity of $U_{P M N S}$. This equation will be referred to as the unitarity condition in this paper. Since this condition always holds, it must be obeyed by any combination of $S_{i}$ and $T$ that generates a full-mixing matrix. As Eq.(8) , it 
can also be written in more explicit forms. Equations (8) and (9) are the starting point of our discussion that follows.

When $\operatorname{Tr}[T]$ is real, $T_{d}$ is given by one of the $T$ matrices in Eq.(1). Then from Eq.(8) it follows that

$$
\begin{aligned}
& \operatorname{Re}\left[w_{i}\right]=1-2\left|\left(U_{P M N S}\right)_{\alpha i}\right|^{2}(1-\cos 2 \pi k / m) \\
& \operatorname{Im}\left[w_{i}\right]=2\left[\left|\left(U_{P M N S}\right)_{\beta i}\right|^{2}-\left|\left(U_{P M N S}\right)_{\gamma i}\right|^{2}\right] \sin 2 \pi k / m
\end{aligned}
$$

where $\beta, \gamma \neq \alpha$ and $\beta<\gamma$. Solutions to Eqs.(10) and (11) should respect the conditions

$$
0 \leq\left|\left(U_{P M N S}\right)_{\rho i}\right|^{2} \leq 1, \quad \rho=\alpha, \beta, \text { or } \gamma
$$

which will be referred to as reality conditions. Explicit expressions for $\left|\left(U_{P M N S}\right)_{\rho i}\right|^{2}$ will be given later. Note that the value of $\alpha$ depends on which $T$ matrix given in Eq.(11) is used in Eq.(8). Using a different $T$ matrix but keeping $m$ and $k$ fixed results in a reordering of the elements in the mixing vector by which we mean a column of the mixing matrix. One may use any $T$ matrix given in Eq.(11) as long as the results are consistent with the experimental data.

Together with the unitarity and the reality conditions, the above formalism provides a simple way to derive mixing matrix or mixing elements. For example, it was shown in [45] that the group $\operatorname{PSL}\left(2, Z_{7}\right)$ can lead to a mixing matrix given by ${ }^{3}$

$$
\left\|U_{P M N S}\right\|=\frac{1}{2}\left(\begin{array}{ccc}
\sqrt{\frac{1}{2}(3+\sqrt{7})} & 1 & \sqrt{\frac{1}{2}(3-\sqrt{7})} \\
1 & \sqrt{2} & 1 \\
\sqrt{\frac{1}{2}(3-\sqrt{7})} & 1 & \sqrt{\frac{1}{2}(3+\sqrt{7})}
\end{array}\right) .
$$

The representation matrices of $P S L\left(2, Z_{7}\right)$ are given by

$$
F=\frac{2}{\sqrt{7}}\left(\begin{array}{ccc}
s_{1} & s_{2} & s_{3} \\
s_{2} & -s_{3} & s_{1} \\
s_{3} & s_{1} & s_{2}
\end{array}\right), \quad G=\frac{2}{\sqrt{7}}\left(\begin{array}{ccc}
e^{4 \pi i / 7} & 0 & 0 \\
0 & e^{2 \pi i / 7} & 0 \\
0 & 0 & e^{8 \pi i / 7}
\end{array}\right)
$$

where $s_{k}=\sin k \pi / 7 . S_{1}, S_{2}$, and $T$ can be chosen as $F, G^{2} F G^{3} F G$, and $G^{3} F$, respectively. The $\left\|U_{P M N S}\right\|$ matrix given in Eq.(13) can be obtained by diagonalizing $S_{i}$ and $T$. The

\footnotetext{
${ }^{3}$ Following [45], we use $\left\|U_{P M N S}\right\|$ to denote the matrix with every entry being the absolute value of the corresponding one in the mixing matrix $U_{P M N S}$.
} 
formalism given above provides a different but more efficient way to derive it. More explicit formalism and examples will be given later.

Now we have all the necessary ingredients, but before proceeding, there are a few comments we would like to make.

1. Since $T \in S U(3)$, if $\operatorname{Tr}[T]$ is not real, then it does not have a +1 eigenvalue and hence no vacuum alignment can break $G_{f}$ into the $\mathbf{Z}_{m}$ symmetry generated by $T$. It can be arranged that residual symmetries are preserved indirectly [46, 55], but it sounds more natural if they emerge directly, and thus, except in an example given in Sec. IV, we assume in most of our discussions that $\operatorname{Tr}[T]$ is real.

2. When degeneracy occurs among the eigenvalues of $T$, whether $\operatorname{Tr}[T]$ is real or not, the lepton mixing matrix cannot be determined unambiguously and the solutions to Eq.(8) are not unique. Nevertheless, the vanishing of $\operatorname{Im}(\operatorname{Tr}[T])$ assures that for any $m>2$, the eigenvalues of $T$ are nondegenerate and the mixing matrix can be determined unambiguously. As to the case where $m=2$, to eliminate the ambiguity, one may enlarge the $\mathbf{Z}_{2}$ symmetry generated by $T$ to a larger one, e.g. a $\mathbf{Z}_{2} \times \mathbf{Z}_{2}$. An example is given in footnote 4 . Since we are interested in general cases, we will not consider this particular case further.

3. Unlike the assumption on $\operatorname{Tr}[T], \operatorname{Tr}\left[W_{i}\right]$ are assumed to be real mostly for calculational simplicity. In many cases, including the $P S L\left(2, Z_{7}\right)$ example discussed above, $\operatorname{Tr}\left[W_{i}\right]$ are not real. But we should mention that this assumption is indeed satisfied by many models leading to well-known mixing patterns (e.g., TBM, BM, and the golden ratio mixings). In addition, it can be treated as a reasonable phenomenological assumption. The reason is that the equalities between the absolute values of mixing elements are still phenomenologically viable, and from Eq.(11) it follows that when Eq.(16) is satisfied, $\operatorname{Im}\left[w_{i}\right]$ vanishes automatically. Detailed discussion about the case where $\operatorname{Tr}\left[W_{i}\right]$ are not real will be presented in Sec. IV.

4. From experimental data, it follows that in the minimal scenario, $\sin \theta_{13}$ can only result from Eq.(15) (see below), and hence, to accommodate measured $\sin \theta_{13} \sim 0.15,1-w_{i}$ must be almost vanishing because $\sin ^{2} \theta_{13} \sim 0.02$, which requires a large $p_{i}$ (recall that $\left.w_{i}=-1-2 \cos 2 \pi n_{i} / p_{i}\right)$ such that $n_{i} / p_{i}$ can be close to $1 / 2$. Numerical computation 
also shows that to fit the data, $p_{i}$ should be larger than 10 . Large $p_{i}$ corresponds to a $S_{i} T$ of large order. Therefore, the larger the $p_{i}$ is, the less the chance for small groups to accommodate the data. Allowing corrections to $\sin \theta_{13}$ may significantly lower the requirement, but introducing large corrections may also require the price of weakening the role of symmetry to be paid. More discussion can be found in Sec. IV.

5. If all $S_{i} \in G_{f}$ and $m \geq 3$, then the matrix $\left\|U_{P M N S}\right\|$ is completely determined by $S_{i}$ and $T$ and is referred to as a full-mixing matrix. In the case where both $\operatorname{Tr}[T]$ and $\operatorname{Tr}\left[S_{i} T\right]$ are real, one can show that Eq.(16) (see below) always leads to a vanishing mixing element or a maximum Dirac phase in an appropriate parametrization [56]. The vanishing element implies that $w_{i}=1$, and thus from Eq.(17), one finds that $p_{i}=2$ and $\left(S_{i} T\right)^{2}=1$, i.e. one $S_{i} T$ must be of order 2 , which can also be seen from the result presented in the next section. Note that $p_{i}$ cannot be completely determined in this way.

\section{MIXING PATTERNS IN THE MINIMAL SCENARIO}

In this section, we concentrate on the full-mixing patterns in the minimal scenario where both $\operatorname{Tr}[T]$ and $\operatorname{Tr}\left[W_{i}\right]$ are real. As discussed above, the residual symmetries are strongly constrained by the unitarity condition Eq.(9). Thanks to Eq.(6), $\operatorname{Tr}[T]$ and $\operatorname{Tr}\left[W_{i}\right]$ depend on the orders of $T$ and $W_{i}$, which can then be solved from Eq.(9). Obviously it admits many solutions. What is interesting and somewhat surprising in the minimal case is that the complete set of solutions can be determined. Consequently, all the possible mixing patterns can also be derived. After some general discussions about the solutions, the one that leads to a golden ratio mixing will be discussed in detail to demonstrate the way to derive the mixing pattern and the flavor symmetry corresponding to a particular solution and clarify its difference from the group theoretical method.

We begin with Eqs.(10) and (11) which determine the mixing elements. When both $\operatorname{Tr}\left[W_{i}\right]$ and $\operatorname{Tr}[T]$ are real, they can be written as [50]

$$
\begin{aligned}
& \left|\left(U_{P M N S}\right)_{\alpha i}\right|^{2}=\frac{1-w_{i}}{4 \sin ^{2} \frac{k \pi}{m}}=\frac{1+\operatorname{Tr}\left[W_{i}\right]}{4 \sin ^{2} \frac{k \pi}{m}} \\
& \left|\left(U_{P M N S}\right)_{\beta i}\right|^{2}=\left|\left(U_{P M N S}\right)_{\gamma i}\right|^{2}=\frac{1}{2}\left(1-\left|\left(U_{P M N S}\right)_{\alpha i}\right|^{2}\right) .
\end{aligned}
$$


We also assume that all $S_{i} \in G_{f}$. Hence the unitarity condition given by Eq.(9) must be respected. As discussed below Eq.(17), if $\operatorname{Tr}\left[W_{i}\right]$ is real, then

$$
\operatorname{Tr}\left[W_{i}\right]=1+2 \cos \frac{n_{i}}{p_{i}} 2 \pi
$$

where $p_{i}$ is the order of $W_{i}$. Note that the greatest common divisor of $n_{i}$ and $p_{i}, \operatorname{gcd}\left(n_{i}, p_{i}\right)=$ 1. Now from Eqs.(2) and (9), one has

$$
1+2 \cos \frac{k}{m} 2 \pi=-\sum_{i=1}^{3} \operatorname{Tr}\left[W_{i}\right]=-3-2 \sum_{i=1}^{3} \cos \frac{n_{i}}{p_{i}} 2 \pi
$$

which can be written as

$$
\sum_{j=1}^{4} 2 \cos \frac{n_{j}}{p_{j}} 2 \pi=-4
$$

where $k$ is replaced by $p_{4}$ and $m$ by $n_{4}$. Without loss of generality, we require that $0<$ $n_{j} / p_{j} \leq 1 / 2$. Equation (19) is a necessary condition that $p_{j}$, the orders of $T$ and $W_{i}$ (or $\left.S_{i} T\right)$ must obey. Together with $n_{i}$, they can be used to construct explicitly the (minimal) group corresponding to a solution. Its relation with the group theoretical method will be discussed shortly.

We find that a complete and rigorous solution to Eq.(19) can be derived by using algebraic number theory [58, 59], which can translate Eq.(19) into a much simpler arithmetic equation. Although a rigorous derivation is interesting by its own right, it is somewhat lengthy because some mathematical concepts and results need to be introduced. In addition, the solutions given below can be verified numerically by evaluating Eq.(19) continuously until $p_{i}$ reach a desired large value. Therefore, in order to concentrate on physical discussion, we will present the detailed mathematical derivation elsewhere.

Since $n_{i}$ can be easily found from Eq.(19) when $p_{i}$ are given, below we denote the solution to Eq.(19) by $\left\{p_{j}\right\}$. Although expected, it is still a little bit surprising to find that, besides obviously disfavored solutions, i.e., $\{1,2,2,2\}$ and $\left\{2,2, p_{3}, p_{4}\right\}$, Eq.(19) admits only three other solutions: $\{3,3,3,3\},\{2,3,3,4\}$, and $\{2,3,5,5\}$. Note that $p_{3}$ and $p_{4}$ in the second solution satisfy $n_{3} / p_{3}+n_{4} / p_{4}=1 / 2$, where $n_{3}$ and $n_{4}$ are arbitrary integers satisfying $0<n_{j} / p_{j} \leq 1 / 2$ and $\operatorname{gcd}\left(n_{j}, p_{j}\right)=1$.

To find the $\left\|U_{P M N S}\right\|$ matrix corresponding to a solution, one must first assign one $p_{j}$ to $m$ and the others to $p_{1,2,3}$ in Eq.(17). It is obvious that the first solution $\{1,2,2,2\}$ does not respect the assumption that $m \geq 3$, and hence it cannot lead to an unambiguous mixing 
matrix. The second solution $\left\{2,2, p_{3}, p_{4}\right\}$ is also phenomenologically disfavored because of the same reason, which requires that any $p_{j}=2$ can only be assigned to the order of an $S_{i} T$. From Eqs.(15) and (17) it follows that $U_{\alpha i}=0$ if $p_{i}=2$. However, $p_{1,2}=2$ results in two vanishing elements which lead to two vanishing mixing angles and hence this solution is not phenomenologically favorable.

The third solution $\left\{p_{i}, m\right\}=\{3,3,3,3\}$ leads to an interesting $\left\|U_{P M N S}\right\|$ matrix in which all the elements equal $1 / \sqrt{3}$, but it is also not phenomenologically favorable because of its large deviation from the experimental data. Note that it leads to a maximum Dirac CP phase.

Therefore, we are left with the last two solutions. From Eqs.(15)-(17), it is easy to show that the assignment $\left\{p_{1}, p_{2}, p_{3}, m\right\}=\{3,3,2,4\},\{4,3,2,3\},\{3,5,2,5\}$, or $\{5,5,2,3\}$ leads to BM, TBM, or the golden ratio mixings, respectively. ${ }^{4}$ As an example, let $\left\{p_{1}, p_{2}, p_{3}, m\right\}=$ $\{3,5,2,5\}$ and $\left\{n_{1}, n_{2}, n_{3}, k\right\}=\{1,2,1,1\}$. From Eq.(17) and $\operatorname{Tr}\left[W_{i}\right]=-w_{i}$, one has

$$
w_{1}=0, \quad w_{2}=-1-2 \cos [4 \pi / 5]=(\sqrt{5}-1) / 2, \quad w_{3}=1 .
$$

Then from Eq.(15) and $4 \sin ^{2} \pi / 5=10 /(5+\sqrt{5})$, it follows that

$$
\left|\left(U_{P M N S}\right)_{\alpha 1}\right|^{2}=\frac{5+\sqrt{5}}{10}, \quad\left|\left(U_{P M N S}\right)_{\alpha 2}\right|^{2}=\frac{5-\sqrt{5}}{10}, \quad\left|\left(U_{P M N S}\right)_{\alpha 3}\right|^{2}=0 .
$$

Other elements can be obtained from Eq.(16), and the resulting $U_{P M N S}$ is a golden ratio mixing matrix (for explicit expression, see [45, 60]). Note that one must set $\alpha=e=$ 1 for phenomenological reasons. Also note that mixing matrices obtained by exchanging $p_{i}$ or using a different value for $\alpha$ are just the original mixing matrix with its rows and columns being reordered. But for the assignments discussed above reordering is obviously phenomenologically unacceptable.

This example shows clearly the difference between the method discussed here and the group theoretical method. The latter requires the knowledge of the flavor group and its representation. Here what we need is a set of $p_{i}$ and $n_{i}$ that satisfy the unitarity condition and

\footnotetext{
${ }^{4}$ As discussed in the last section, we require that $m \neq 2$. If $T^{2}=\mathbb{I}$, to fix the mixings, one may enlarge the $\mathbf{Z}_{2}$ symmetry generated by $T$ to a larger one. For example, if $\{3,5,5,2\}$ is assigned to $\left\{p_{i}, m\right\}$ and $G_{e}$ is chosen to be $\mathbf{Z}_{2} \times \mathbf{Z}_{2}$, then $G_{e}$ contains two $\mathbf{Z}_{2}$ generators, each of which determines a row of $U$. Therefore, $U$ can also be completely determined in this case. The corresponding mixing matrix can be found in [45].
} 
no prior knowledge of the flavor group is needed, which is to be determined by the solution. For instance, the example discussed above corresponds to the solution $\left\{p_{i}\right\}=\{2,3,5,5\}$ which indicates that the flavor group must contain $\mathbf{Z}_{2}, \mathbf{Z}_{3}, \mathbf{Z}_{5}$, and $\mathbf{Z}_{2} \times \mathbf{Z}_{2}$ subgroups and its order must be a multiple of 60 . It is not hard to find that the minimal finite group satisfying this condition is $\mathbf{A}_{5}$. One can use $p_{i}$ and $n_{i}$ given above to verify that this solution can indeed be realized by $\mathbf{A}_{5}$. Similarly, from the solution leading to TBM, one can show that $\mathbf{S}_{4}$ is the minimal horizontal symmetry that can produce TBM as a full-mixing matrix, which was first pointed out in [51]. In addition, one may also use $p_{i}$ and $n_{i}$ to construct the group. In summary, the solution associated with a mixing matrix can be regarded as the necessary conditions or minimal requirements for a flavor symmetry to produce the mixing matrix. Although necessary conditions are not sufficient to establish the existence of a finite group, so far no counterexample has been found.

In the discussion above it is assumed that all $S_{i}$ and $T$ belong to $G_{f}, \operatorname{Tr}\left[S_{i} T\right]$ and $\operatorname{Tr}[T]$ are real and the order of $T$ is larger than 2. As discussed in the previous section, although not mandatory, they are general and reasonable assumptions on residual symmetries. It is interesting to see that phenomenologically viable mixing patterns can lead to include only several well-known ones. This result provides a different way to understand those mixing patterns as natural consequences of discrete flavor symmetries. On the other hand, the no-go result obtained above implies that, to accommodate the mixing data including $\sin \theta_{13}$, which is small but far from vanishing, some of the assumptions adopted in this section may have to be relaxed, which will be discussed in the next section.

\section{BEYOND THE MINIMAL SCENARIO}

In this section, we focus on the consequences of allowing $\operatorname{Tr}\left[S_{i} T\right]$ or $\operatorname{Tr}\left[W_{i}\right]$ to be complex valued. In this case, the eigenvalues of $W_{i}$ satisfying $\lambda^{p_{i}}=1$ can be written as $e^{2 \pi i n_{i} / p_{i}}$, $e^{2 \pi i m_{i} / p_{i}}$ and $e^{-2 \pi i\left(n_{i}+m_{i}\right) / p_{i}}$. Without loss of generality, we require that $0 \leq n_{i}, m_{i}<p_{i}$, 
$n_{i}+m_{i} \neq 0$, and $\operatorname{gcd}\left(n_{i}, p_{i}\right)=1$. The solutions to Eqs.(10) and (11) are given by

$$
\begin{aligned}
\left|\left(U_{P M N S}\right)_{\alpha i}\right|^{2}= & \left(\sin ^{2} \frac{k \pi}{m}\right)^{-1} \cos \left[\frac{n_{i}}{2 p_{i}} 2 \pi\right] \cos \left[\frac{m_{i}}{2 p_{i}} 2 \pi\right] \cos \left[\frac{n_{i}+m_{i}}{2 p_{i}} 2 \pi\right], \\
\left|\left(U_{P M N S}\right)_{\beta i}\right|^{2}= & -\left(2 \sin ^{2} \frac{k \pi}{m} \cos \frac{k \pi}{m}\right)^{-1} \cos \left[\left(\frac{k}{2 m}+\frac{n_{i}}{2 p_{i}}\right) 2 \pi\right] \\
& \cos \left[\left(\frac{k}{2 m}+\frac{m_{i}}{2 p_{i}}\right) 2 \pi\right] \cos \left[\left(\frac{k}{2 m}-\frac{n_{i}+m_{i}}{2 p_{i}}\right) 2 \pi\right], \\
\left|\left(U_{P M N S}\right)_{\gamma i}\right|^{2}= & -\left(2 \sin ^{2} \frac{k \pi}{m} \cos \frac{k \pi}{m}\right)^{-1} \cos \left[\left(\frac{k}{2 m}-\frac{n_{i}}{2 p_{i}}\right) 2 \pi\right] \\
& \cos \left[\left(\frac{k}{2 m}-\frac{m_{i}}{2 p_{i}}\right) 2 \pi\right] \cos \left[\left(\frac{k}{2 m}+\frac{n_{i}+m_{i}}{2 p_{i}}\right) 2 \pi\right] .
\end{aligned}
$$

One can verify that Eqs.(15) and (16) are recovered when $n_{i}$ or $m_{i}$ vanishes or $n_{i}+m_{i}=p_{i}$.

If all $S_{i} \in G_{f}$, as in the previous section, the unitarity condition must be respected and then from Eq. (9), one has

$$
\sum_{i=1}^{3} V_{i}+2 \cos \alpha_{k m}=-1, \quad V_{i}=\cos \alpha_{n_{i} p_{i}}+\cos \alpha_{m_{i} p_{i}}+\cos \left(\alpha_{n_{i} p_{i}}+\alpha_{m_{i} p_{i}}\right)
$$

and

$$
\sum_{i=1}^{3} V_{i}^{\prime}=0, \quad V_{i}^{\prime}=\sin \alpha_{n_{i} p_{i}}+\sin \alpha_{m_{i} p_{i}}-\sin \left(\alpha_{n_{i} p_{i}}+\alpha_{m_{i} p_{i}}\right)
$$

where $\alpha_{k m}=2 \pi k / m, \alpha_{n_{i} p_{i}}=2 \pi n_{i} / p_{i}$, etc. Unlike the minimal scenario where $\operatorname{Tr}\left[W_{i}\right]$ are real, the above two equations are much more involved, and the complete set of solutions can hardly be obtained in this case. But, as in the minimal scenario, solutions for reasonably large $p_{i}$ can be exhausted by numerical calculations. Besides that, they are also useful for one to approach possible solutions quickly, especially when some $p_{i}$ are given.

As an example, let $m=4$ and $p_{1}=12$. Since $\cos \left(2 \pi n_{1} / 12\right)= \pm \sqrt{3} / 2, V_{1}$ and $V_{1}^{\prime}$ are expected to be a quadratic number ${ }^{5}$ of the form $a+b \sqrt{3}$ except for particular choices of $n_{1}$ and $m_{1}$ [e.g., $n_{1}=1$ and $m_{1}=5$ or 6 , which lead to $\left|\left(U_{P M N S}\right)_{11}\right|=0$ ]. Therefore, to satisfy Eq.(23) , it is reasonable to require that $p_{2}$ or $p_{3}$ also gives rise to some quadratic terms with the factor $\sqrt{3}$. The simplest choice is to let, e.g. $p_{3}=12$. Then, without much effort, we

\footnotetext{
${ }^{5}$ By quadratic number we mean a number satisfying a quadratic equation with rational coefficients.
} 
find that setting $p_{2}=3$ can lead to the mixing matrix

$$
\left\|U_{P M N S}\right\|=\frac{1}{2 \sqrt{2}}\left(\begin{array}{ccc}
\sqrt{3+\sqrt{3}} & \sqrt{2} & \sqrt{3-\sqrt{3}} \\
\sqrt{2} & 2 & \sqrt{2} \\
\sqrt{3-\sqrt{3}} & \sqrt{2} & \sqrt{3+\sqrt{3}}
\end{array}\right) .
$$

which presumably can be realized by the group $\boldsymbol{\Delta}(432)$.

Although the resulting $\sin ^{2} \theta_{13}=0.158$ does not agree with the data well, the point shown by this example is that if any $p_{i}$ grows large, especially when the value of $\cos \left(2 \pi n_{i} / p_{i}\right)$ or $\sin \left(2 \pi n_{i} / p_{i}\right)$ is not a rational number, ${ }^{6}$ then for generic $n_{i}$ and $m_{i}$, it often occurs that another $p_{i}$ has to acquire the same value. This also explains to some extent why identical columns up to permutations often show up in full-mixing matrices, as the one given above. Moreover, if $m$ takes a value leading to a nonrational $\cos (2 \pi k / m)$, e.g. $m=5$, 7 , etc. the above unitary conditions are even harder to be satisfied since $m$ appears only in one of them, i.e., Eq.(23). This implies that even a large group can lead to only a rather limited number of mixing patterns. A slight improvement can be achieved by allowing $\operatorname{Tr}[T]$ to be complex, which will be discussed later in this section.

As another interesting application, consider $m=3$ corresponding to $G_{e}=\mathbf{Z}_{3}$ which occurs frequently. When $m=3$, one finds that Eqs. (20) $-(22)$ can be written as

$$
\left|\left(U_{P M N S}\right)_{\rho i}\right|^{2}=\frac{4}{3} \cos \left[\frac{n_{\rho i}}{2 p_{\rho i}} 2 \pi\right] \cos \left[\frac{m_{\rho i}}{2 p_{\rho i}} 2 \pi\right] \cos \left[\frac{n_{\rho i}+m_{\rho i}}{2 p_{\rho i}} 2 \pi\right]
$$

where $\rho=\alpha, \beta$, or $\gamma, p_{\alpha i}=p_{i}, \quad n_{\alpha i}=n_{i}, m_{\alpha i}=m_{i}$, and

$$
\begin{aligned}
\frac{n_{\beta i}}{2 p_{\beta i}} & =\frac{1}{6}+\frac{n_{i}}{2 p_{i}}, \quad \frac{m_{\beta i}}{2 p_{\beta i}}=\frac{1}{6}+\frac{m_{i}}{2 p_{i}}, \\
\frac{n_{\gamma i}}{2 p_{\gamma i}} & =\frac{1}{6}-\frac{n_{i}}{2 p_{i}}+H\left(\frac{n_{i}}{2 p_{i}}-\frac{1}{6}\right) \\
\frac{m_{\gamma i}}{2 p_{\gamma i}} & =\frac{1}{6}-\frac{m_{i}}{2 p_{i}}+H\left(\frac{m_{i}}{2 p_{i}}-\frac{1}{6}\right)
\end{aligned}
$$

\footnotetext{
${ }^{6}$ A more precise condition can be given in terms of the algebraic degree of $\cos \left(2 \pi n_{i} / p_{i}\right)$ or $\sin \left(2 \pi n_{i} / p_{i}\right)$, which is the lowest degree of the algebraic equations with rational coefficients it satisfies. Except for $m=1$ or 2 , the algebraic degree of $\cos (2 \pi n / m)$ is given by $\varphi(m) / 2[61]$ where $\varphi(m)$ is Euler's $\varphi$-function or Euler's totient function [62]. For example, for $m=3,4,6, \varphi(m) / 2=1$ and hence $\cos (2 \pi n / m)$ is a rational number of degree one. For $m=5,8,10$ and $12, \varphi(m) / 2=2$ and $\cos (2 \pi n / m)$ is a quadratic number of degree two. The degrees of numbers involved in algebraic operations, including addition, product, division, etc. can provide valuable information about the outcomes of these operations.
} 
in which $p_{\beta i}$ and $p_{\gamma i}$ are chosen to be the smallest (positive) integers satisfying the above equations and $H(x)$ is the unit step function defined as $H(x)=1$ (for $x>0$ ) and $H(x)=0$ (for $x<0$ ). As shown by Eq.(25) , the similarity among the expressions for $\left|\left(U_{P M N S}\right)_{\rho i}\right|^{2}$ indicates that $p_{\beta i}$ and $p_{\gamma i}$ can also play the role of $p_{i}$. Therefore, by assigning $p_{i}, p_{\beta i}$ and $p_{\gamma i}$ to the orders of $S_{i} T$, one can construct a mixing matrix with identical columns and rows up to permutations. For example, $\left\{p_{3}, n_{3}, m_{3}, m, k\right\}=\{5,2,3,3,1\}$ results in $p_{\beta i}=p_{\gamma i}=15$ and the squared mixing vector $(6-2 \sqrt{5}, 3+\sqrt{5}, 3+\sqrt{5}) / 12$. Then one may let $p_{1,2}=15$ and the mixing matrix

$$
\left\|U_{P M N S}\right\|=\frac{1}{2 \sqrt{6}}\left(\begin{array}{ccc}
\sqrt{5}+1 & \sqrt{5}+1 & \sqrt{10}-\sqrt{2} \\
\sqrt{10}-\sqrt{2} & \sqrt{5}+1 & \sqrt{5}+1 \\
\sqrt{5}+1 & \sqrt{10}-\sqrt{2} & \sqrt{5}+1
\end{array}\right)
$$

can be obtained by permuting the elements in the third column. A possible issue of the mixing patterns being alike is that to fit the experimental data, somewhat large corrections are required since in every column there is an element equal to $\left|\left(U_{P M N S}\right)_{e 3}\right|$. Also note that in this example $\operatorname{Tr}\left[W_{3}\right]$ is real but $\operatorname{Tr}\left[W_{1,2}\right]$ are not. In general, to produce mixing matrices like the one above, some $\operatorname{Tr}\left[W_{i}\right]$ must be complex valued; otherwise, the matrix $\left\|U_{P M N S}\right\|$ should have two identical rows.

We now turn to phenomenological implications. As we know, most known full-mixing patterns cannot fit the mixing data exactly within the experimentally allowed range. Nevertheless, by using the method discussed above, it is not hard to find one. For example, $\left\{p_{1}, p_{2}, p_{3}, m\right\}=\{20,3,10,3\}$ can lead to

$$
\|U\|=\frac{1}{2 \sqrt{6}}\left(\begin{array}{ccc}
\sqrt{9+\sqrt{5}+c} & 2 \sqrt{2} & \sqrt{7-\sqrt{5}-c} \\
\sqrt{6-2 \sqrt{5}} & 2 \sqrt{2} & \sqrt{10+2 \sqrt{5}} \\
\sqrt{9+\sqrt{5}-c} & 2 \sqrt{2} & \sqrt{7-\sqrt{5}+c}
\end{array}\right)
$$

where $c=\sqrt{6(5-\sqrt{5})}$. The mixing angles can be extracted as follows: $\sin ^{2} \theta_{13}=0.029$, $\sin ^{2} \theta_{23}=0.62$, and $\sin ^{2} \theta_{12}=0.34$. The obvious problem with this example is that it requires a large group, presumably $\boldsymbol{\Delta}(600)$. In fact, this result is confirmed by group theoretical calculation in a recent work [54] in which a scan of groups with orders up to 1536 is performed. Our calculation does not need any prior knowledge about $\boldsymbol{\Delta}(600)$ and, as discussed above, it is the solution that suggests $\boldsymbol{\Delta}(600)$ as a candidate. Besides $\boldsymbol{\Delta}(600)$, the other two mixing 
patterns found in [54] for $\boldsymbol{\Delta}(1536)$ and $\left(\mathbf{Z}_{18} \times \mathbf{Z}_{6}\right) \rtimes \mathbf{S}_{3}$ can also be obtained from Eqs. (20])(22). Just in case it is needed, below we give the exact value of the mixing matrix for $\Delta(1536)$

$$
\|U\|=\frac{1}{\sqrt{6}}\left(\begin{array}{ccc}
\sqrt{2+\sqrt{2+\sqrt{2}}} & \sqrt{2} & \sqrt{2-\sqrt{2+\sqrt{2}}} \\
\sqrt{2-c_{-}} & \sqrt{2} & \sqrt{2+c_{-}} \\
\sqrt{2-c_{+}} & \sqrt{2} & \sqrt{2+c_{+}}
\end{array}\right)
$$

where $c_{ \pm}=\sqrt{2 \pm \sqrt{2 \mp \sqrt{3}}}$. The numerical values of $\sin ^{2} \theta_{i j}$ can be found in [54]. As to the group $\left(\mathbf{Z}_{18} \times \mathbf{Z}_{6}\right) \rtimes \mathbf{S}_{3}$, the corresponding mixing matrix does not admit an expression in terms of exact values as the one shown above ${ }^{7}$.

Besides [54], it is also reported in [53] that no $S U(3)$ subgroup of order less than 512 can produce the full-mixing data. For us this is almost an expected result since from Eqs.(20)(22) it is easy to see that small $\sin \theta_{13}$ requires one or more angles involved to be close to $\pi / 2$ or $3 \pi / 2$, and hence, together with the reality conditions, it would require $p_{i}$ or $m$ to be large. Equations (20) $-(22)$ can also be evaluated numerically and the result agrees with them, as expected. Since in general, mixing parameters also receive contributions from higher-order corrections including radiative corrections, this result implies that it might be more plausible to introduce sizable corrections (or free parameters) to the mixing patterns obtained from discrete symmetries, which can be considered as leading order contributions [43]. Partial mixing is also a reasonable option, as discussed in [50, 52].

Finally, we comment on the case where $\operatorname{Im}(\operatorname{Tr}[T]) \neq 0$. When it occurs, the unitarity conditions given by Eqs.(23) and (24), which follow from Eq.(9), should be modified to

$$
\sum_{i=1}^{4} V_{i}=0, \quad V_{i}=\cos \alpha_{n_{i} p_{i}}+\cos \alpha_{m_{i} p_{i}}+\cos \left(\alpha_{n_{i} p_{i}}+\alpha_{m_{i} p_{i}}\right)
$$

\footnotetext{
${ }^{7}$ Using algebraic number theory, one can show that mixing elements can be expressed in terms of exact values only when some conditions are satisfied. For example, in the case where $m=3$, the orders of $S_{i} T$ must be products of Fermat numbers. By exact values we mean numbers that can be expressed in terms of rational numbers involving only sums, products and square roots, such as $(2-\sqrt{3}) / 3$. This also implies that among all the mixing patterns that can be expressed in exact forms, only very particular ones such as those discussed in this paper, can be produced by discrete symmetries. Therefore, one can show that some mixing patterns, e.g. the hexagonal mixing, cannot be produced by discrete symmetries at least in the framework discussed in this work. More detailed discussion will be given elsewhere.
} 
and

$$
\sum_{i=1}^{3} V_{i}^{\prime}=V_{4}^{\prime}, \quad V_{i}^{\prime}=\sin \alpha_{n_{i} p_{i}}+\sin \alpha_{m_{i} p_{i}}-\sin \left(\alpha_{n_{i} p_{i}}+\alpha_{m_{i} p_{i}}\right) .
$$

Although more complicated and even harder to be satisfied, it allows new mixing patterns for particular $p_{i}$. As an example, let $m=p_{4}=7, n_{4}=1$. The quickest way to find a solution in this case is to use the trick of assigning the value of $p_{4}$ or $m$ to another $p_{i}$, which fortunately works again. After that, the remaining $p_{i}$ can be easily derived. One can verify that a solution can be found and it leads to another $P S L\left(2, Z_{7}\right)$ mixing matrix given in [45] [see Eq. (40) there].

Although a solution is found in the example above, it is not hard to see that it is very likely that this solution is the only solution for the case where $m=7$, which shows that if $m$ is associated with a $\cos (2 \pi / m)$ with a high degree (see footnote 6 ), the unitary conditions are hard to be satisfied and solutions may not always exist. It also provides further support to our finding that using large groups may not help too much for the purpose of producing full-mixing matrices that can fit all the experimental data. One may wonder whether good chances would appear if the restriction to $S U(3)$ is removed. However, it does not seem to be very likely because if $\operatorname{det} T \neq 1$, then $p_{i}$ are constrained not only by the unitarity condition which will become more complicated, but also by the relation Eq.(6). Since this is the case beyond the scope of this paper, we will leave it for future work.

\section{CONCLUSIONS}

As shown in the previous sections, the method discussed in this paper can provide an efficient way to derive neutrino mixing patterns determined by underlying discrete flavor symmetries. Although most of our attention is paid to full-mixing patterns, the formalism developed in this paper can also be used to calculate mixing elements in the partial-mixing case. It can be used to cross-check with group theoretical calculations or as a simpler way to reach some known results. It also gives the necessary conditions that can lead to the flavor symmetry corresponding to a mixing matrix or mixing-matrix column derived by this method. Since the properties of most $S U(3)$ subgroups can be found in existing literatures, e.g. [63 65], one may find an appropriate group without much trouble in most cases and the physical viability can be verified concretely, as shown by the $\boldsymbol{\Delta}(600)$ example.

Moreover, it can also shed new light on the relation between neutrino mixing and discrete 
symmetries. We find that in the minimal scenario where some general assumptions are adopted, except phenomenologically disfavored mixing patterns, discrete symmetries cannot lead to mixing patterns other than the well-known TBM, BM and golden ratio mixings. This result not only provides a new way to understand these mixing patterns as simple and natural consequences of discrete symmetries, but also clearly indicates the limitation of the minimal scenario and how to go beyond it. Another interesting result can be found by this method is that, although relaxing some assumptions can lead to new mixing patterns, to fit the mixing data, relative large groups are required for full-mixing matrices unless sizable corrections are allowed.

\section{Acknowledgements}

This work was supported in part by the National Science Foundation of China (NSFC) under the Grant No. 10965003.

[1] G. Altarelli, arXiv:1210.3467 [hep-ph].

[2] A. Y. .Smirnov, arXiv:1210.4061 [hep-ph].

[3] D. V. Forero, M. Tortola, and J. W. F. Valle, Phys. Rev. D 86 (2012) 073012 arXiv:1205.4018 [hep-ph]].

[4] G. Fogli et. al., Phys. Rev. D 86 (2012) 013012 [arXiv:1205.5254 [hep-ph]].

[5] M. C. Gonzalez-Garcia, M. Maltoni, J. Salvado, T. Schwetz, arXiv:1209.3023 [hep-ph].

[6] G. Altarelli, F. Feruglio and L. Merlo, arXiv:1205.5133 [hep-ph].

[7] A. Y. .Smirnov, J. Phys. Conf. Ser. 335 (2011) 012006 arXiv:1103.3461 [hep-ph]].

[8] S. -F. Ge, D. A. Dicus and W. W. Repko, Phys. Lett. B 702 (2011) 220.

[9] S. -F. Ge, D. A. Dicus and W. W. Repko, Phys. Rev. Lett. 108 (2012) 041801.

[10] H. Ishimori and T. Kobayashi, Phys. Rev. D 85 (2012) 125004 arXiv:1201.3429 [hep-ph]].

[11] S. Dev, R. R. Gautam, and L. Singh, Phys. Lett. B 284 (2012) 708 [arXiv:1201.3755 [hep-ph]].

[12] H.-J. He and X.-J. Xu, Phys. Rev. D 86 (2012) 111301 [arXiv:1203.2908 [hep-ph]].

[13] C. Hagedorn and D. Meloni, Nucl. Phys. B 862 (2012) 691 [arXiv:1204.0715 [hep-ph]].

[14] H. Ishimori and E. Ma, Phys. Rev. D 86 (2012) 045030 arXiv:1205.0075 [hep-ph]]. 
[15] C. Liu and Z. -h. Zhao, arXiv:1205.3849.

[16] C. Liu, Commun. Theor. Phys. 47 (2007) 1088 hep-ph/0507298.

[17] G. Altarelli, F. Feruglio, L. Merlo and E. Stamou, JHEP 1208 (2012) 021 [arXiv:1205.4670 [hep-ph]].

[18] E. I. Lashin, M. Abbas, N. Chamoun and S. Nasri, Phys. Rev. D 86 (2012) 033013 arXiv:1206.4754 [hep-ph]].

[19] C. Alvarado, A. Aranda, O. Corradini, A. D. Rojas and E. Santos-Rodriguez, Phys. Rev. D 86 (2012) 036010 arXiv:1206.5216.

[20] Z.-h. Zhao, Phys. Rev. D 86 (2012) 096010 [arXiv:1207.2545 [hep-ph]].

[21] J. A. Acosta, A. Aranda, M. A. Buen-Abad, A. D. Rojas, arXiv:1207.6093.

[22] P. M. Ferreira, W. Grimus, L. Lavoura and P. O. Ludl, JHEP 1209 (2012) 128 arXiv:1206.7072 [hep-ph]].

[23] Y. H. Ahn, S. Baek and P. Gondolo, Phys. Rev. D 86 (2012) 053004 [arXiv:1207.1229 [hep-ph]].

[24] Y. BenTov, X. -G. He and A. Zee, arXiv:1208.1062 [hep-ph].

[25] R. N. Mohapatra and C. C. Nishi, Phys. Rev. D 86 (2012) 073007 [arXiv:1208.2875 [hep-ph]].

[26] E. Ma, arXiv:1209.3374 [hep-ph].

[27] C. Luhn, K. M. Parattu and A. Wingerter, arXiv:1210.1197 [hep-ph].

[28] M.-C. Chen, J. Huang, J.-M. O'Bryan, A. M. Wijangco and F. Yu, arXiv:1210.6982 [hep-ph].

[29] G. -J. Ding, S. Morisi and J. W. F. Valle, arXiv:1211.6506 [hep-ph].

[30] R. Krishnan, P. F. Harrison and W. G. Scott, arXiv:1211.2000 [hep-ph].

[31] M. Holthausen, M. Lindner and M. A. Schmidt, arXiv:1211.5143 [hep-ph].

[32] P. F. Harrison, D. H. Perkins and W. G. Scott, Phys. Lett. B 530 (2002) 167 hep-ph/0202074;

P. F. Harrison and W. G. Scott, Phys. Lett. B 535 (2002) 163 hep-ph/0203209.

[33] F. Vissani, hep-ph/9708483; V. D. Barger, S. Pakvasa, T. J. Weiler and K. Whisnant, Phys. Lett. B 437 (1998) 107 hep-ph/9806387.

[34] T2K Collaboration, K. Abe et. al., Phys. Rev. Lett. 107 (2011) 041801 arXiv:1106.2822 [hep-ex]].

[35] MINOS Collaboration, P. Adamson et. al., Phys. Rev. Lett. 107 (2011) 181802 arXiv:1108.0015 [hep-ex]].

[36] DOUBLE-CHOOZ Collaboration, Y. Abe et. al., Phys. Rev. D 86 (2012) 052008 arXiv:1207.6632 [hep-ex]]. 
[37] RENO Collaboration, J. K. Ahn et. al., Phys. Rev. Lett. 108 (2012) 191802 [arXiv:1204.0626 [hep-ex]].

[38] DAYA-BAY Collaboration, F. P. An et. al., Phys. Rev. Lett. 108 (2012) 171803 arXiv:1203.1669 [hep-ex]].

[39] S. Antusch, Talk given at the 36th International Conference on High Energy Physics,. Melbourne, Australia, July 4-11, 2012.

[40] C. H. Albright, arXiv:0905.0146 [hep-ph].

[41] S. -F. Ge, H. -J. He and F. -R. Yin, JCAP 1005017 (2010) arXiv:1001.0940.

[42] H. -J. He and F. -R. Yin, Phys. Rev. D 84 (2011) 033009 arXiv:1104.2654].

[43] Z. -Z. Xing, Chin. Phys. C 36 (2012) 281 [arXiv:1203.1672 [hep-ph]].

[44] S. Luo and Z.-z. Xing, Int. J. Mod. Phys. A 27 (2012) 1230031 arXiv:1211.4331 [hep-ph]].

[45] R. de Adelhart Toorop, F. Feruglio and C. Hagedorn, Nucl. Phys. B 858 (2012) 437 arXiv:1112.1340 [hep-ph]].

[46] G. -J. Ding, Nucl. Phys. B 862 (2012) 1 [arXiv:1201.3279 [hep-ph]].

[47] S. F. King, C. Luhn and A. J. Stuart, Nucl. Phys. B 867 (2013) 203 [arXiv:1207.5741 [hep-ph]].

[48] W. Grimus, L. Lavoura and P. O. Ludl, J. Phys. G 36 (2009) 115007 arXiv:0906.2689 [hep$\mathrm{ph}]$.

[49] C. S. Lam, Phys. Rev. D 83 (2011) 113002 arXiv:1104.0055 [hep-ph]].

[50] D. Hernandez and A. Y. .Smirnov, Phys. Rev. D 86 (2012) 053014 [arXiv:1204.0445 [hep-ph]].

[51] C. S. Lam, Phys. Rev. Lett. 101 (2008) 121602 arXiv:0804.2622 [hep-ph]]; C. S. Lam, Phys. Rev. D 78 (2008) 073015 [arXiv:0809.1185 [hep-ph]].

[52] D. Hernandez and A. Y. .Smirnov, arXiv:1212.2149.

[53] C. S. Lam, arXiv:1208.5527 [hep-ph].

[54] M. Holthausen, K. S. Lim and M. Lindner, arXiv:1212.2411.

[55] G. -J. Ding and D. Meloni, Nucl. Phys. B 855 (2012) 21 arXiv:1108.2733 [hep-ph]].

[56] H. Fritzsch and Z. -Z. Xing, Prog. Part. Nucl. Phys. 45 (2000) 1 hep-ph/9912358].

[57] Y. Zhang, X. Zhang and B.-Q. Ma, Phys. Rev. D 86 (2012) 093019 [arXiv:1211.3198 [hep-ph]].

[58] H. Pollard and H. G. Diamond, "The Theory of Algebraic Numbers", 3rd rev. edition, Dover Publications, 1998.

[59] S. Lang, "Algebra", rev. 3rd edition, Springer, 2002.

[60] L. L. Everett and A. J. Stuart, Phys. Rev. D 79 (2009) 085005 arXiv:0812.1057]; F. Feruglio 
and A. Paris, JHEP 1103 (2011) 101 arXiv:1101.0393]; G. -J. Ding, L. L. Everett, A. J. Stuart, Nucl. Phys. B 857 (2012) 219 arXiv:1110.1688.

[61] W. Watkins and J. Zeitlin, The American Mathematical Monthly, Vol. 100, No. 5 (1993) 471.

[62] G. H. Hardy and E. M. Wright, "An introduction to the Theory of Numbers", 6th edition, Oxford University Press, 2008.

[63] W. Grimus and P. O. Ludl, J. Phys. A 45 (2012) 233001 arXiv:1110.6376 [hep-ph]].

[64] H. Ishimori et. al.,, Prog. Theor. Phys. Suppl. 183 (2010) 1 arXiv:1003.3552 [hep-th]].

[65] P. O. Ludl, J. Phys. A 43 (2010) 395204 arXiv:1006.1479 [math-ph]]; Erratum-ibid. J. Phys. A 44 (2011) 139501. 